\title{
Giant Anterior Cervical Osteophyte Leading to Dysphagia
}

\author{
Jin Seop Hwang, Chung Kee Chough, Won II Joo \\ Department of Neurosurgery, Yeouido St. Mary's Hospital, Catholic University of Korea
}

\begin{abstract}
Large anterior cervical osteophytes can occur in degeneration of the cervical spine or in diffuse idiopathic skeletal hyperostosis (DISH). Large osteophytes can produce otolaryngological symptoms such as dysphagia, dysphonia, and foreign body sensation. We describe a DISH patient with giant anterior cervical osteophyte causing chronic dysphagia and dysphonia. A 56-year-old man presented with increasing dysphagia, dysphonia, neck pain and neck stiffness. Physical examination of the neck showed a non-tender and hard mass on the left side at the level of C4-5. Radiography showed extensive ossification of anterior longitudinal ligament along the left anterolateral aspect of vertebral bodies from C2 to T1. The ossification was espe cially prominent at the level of $\mathrm{C} 4-5$ and linear breakage was noted at same level. Esophagogram revealed a filling defect along the pharynx and lateral displacement of the esophagus. Giant anterior cervical osteophyte was removed through the leftsided anterolateral cervical approach to the spine. Anterior cervical interbody fusion at C4-5 was followed by posterior cervical fixation using lateral mass screws from C3 to C6. After surgery, dysphagia and dysphonia improved immediately. One year later, cervical CT showed bone fusion at C4-5 bodies and no recurrence of osteophyte. DISH is a common cause of anterior cervical osteophyte leading to progressive dysphagia. Keeping this clinical entity in the differential diagnosis is important in patients with progressive neck stiffness, dysphagia or dysphonia. And surgical treatment of symptomatic anterior cervical osteophyte due to DISH should be considered with a solid fusion procedure preventing postoperative instability or osteophyte progress.
\end{abstract}

Key Words: Anterior cervical osteophyte $\cdot$ Diffuse idiopathic skeletal hyperostosis $\cdot$ Dysphagia $\cdot$ Dysphonia

\section{INTRODUCTION}

Anterior cervical osteophyte can occur in degeneration of the cervical spine or in diffuse idiopathic skeletal hyperostosis (DISH), also known as Forestier's disease ${ }^{11)}$. DISH is a common but underdiagnosed systemic condition that leads to ossification of ligaments and enthuses of the spine as well as peripheral skeleton ${ }^{10)}$. Diagnostic criteria for DISH include flowing ossification along the anterolateral aspect of at least four contiguous vertebrae without obvious signs of the involved intervertebral or apophyseal degenerative changes, and the absence of facet-joint ankylosis. Cervical spine alterations are less frequently encountered in DISH compared to the lumbar or thoracic spine. Although rare, patients with anterior cervical osteophytes may complain of dysphagia especially when

- Received: July 19, 2013 • Revised: September 11, 2013

- Accepted: September 13, 2013

Corresponding Author: Won II Joo, MD

Department of Neurosurgery, Yeouido St. Mary's Hospital, The Catholic University of Korea, \#62 Yeouido-dong, Yeongdeungpo-gu, Seoul

150-713, Korea

Tel: +82-2-3779-2248, Fax: +82-2-786-5809

E-mail: joowonil4858@gmail.com the osteophytes are extraordinarily large ${ }^{5)}$. Giant anterior cervical osteophyte can compress the pharyngoesophageal and the laryngotracheal segments and result in dysphagia, although dysphagia and/or dyspnea resulting from DISH of the cervical spine are rare ${ }^{1}$. Here we present a case report describing a DISH patient with a giant anterior cervical osteophyte causing chronic dysphagia.

\section{CASE REPORT}

A 56-year-old man presented with increasing dysphagia, dysphonia, moderate neck pain and neck stiffness. The patient had no history of trauma to the neck. Physical examination of the neck showed a non-tender and hard mass on the left side at the level of C4-5. Neck motion was decreased. And neurologic examination was normal. Routine blood tests were also normal. Plain radiographs of the cervical spine showed extensive ossification at the anterior longitudinal ligament along the left anterolateral aspect of the vertebral bodies from C2 to T1, especially prominent at C4-5, suggesting DISH. Linear breakage of the ossification is noted at C4-5 (Fig. 1A). In radiographs of the thoracic and lumbar spine, multiple bony 
spurs were identified, too. There was no definite radiographic instability on the cervical dynamic view. Cervical computed tomography (CT) confirmed the giant anterior osteophyte (Fig. 1B). Barium contrast swallow cinematography revealed a filling defect along the pharynx with lateral displacement of the esophagus due to giant anterior osteophyte (Fig. 1C).

The patient underwent surgery with a left-sided anterolateral cervical approach to the spine. The prevertebral fascia was separated and the trachea and esophagus were retracted on the right side, with the carotid sheath on the left side. The C4-5 level was exposed. The giant anterior osteophyte was removed with rongeurs and a high speed drill until the anterior spinal surface from C3 to C6 was flat. All cervical segments except C4-5 were fused due to DISH. We perceived the C4-5 segment to be unstable. So we removed the disc of C4-5 and performed C4-5 interbody fusion using an allograft mixed demineralized bone matrix (DBM) and anterior cervical plate. After several days, we performed posterior screw fixations from the C3 level to the C6 level. A postoperative lateral plain cervical spine radiograph demonstrated removal of the anterior cervical osteophyte at C4-5 (Fig. 2A).

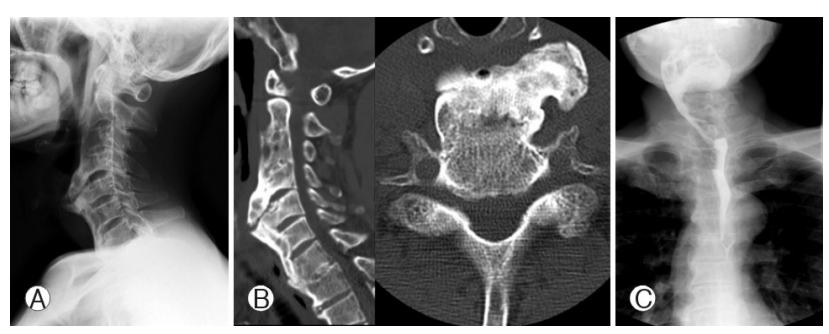

Fig. 1. (A) Plain X-ray shows prominent anterior cervical osteophyte at C4-5. (B) Computed tomographic (CT) scan of the cervical spine reveals extensive ossification of the anterior longitudinal ligament and linear breakage of the ossification is noted at C4-5. (C) Esophagography reveals filling defect along the pharynx due to the ossified anterior longitudinal ligament with lateral displacement of the esophagus.
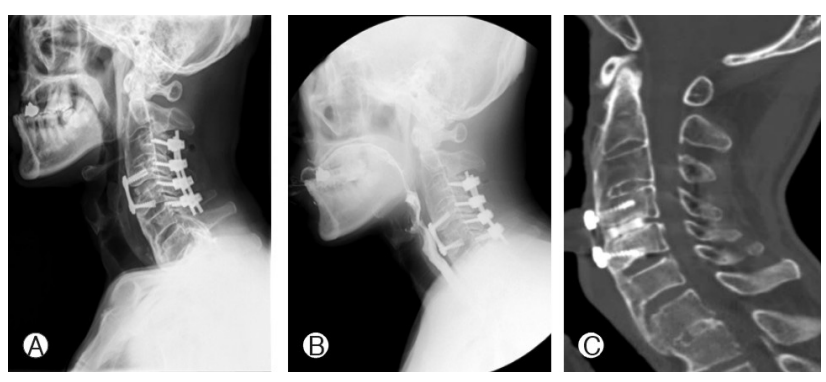

Fig. 2. (A) Postoperative $X$-rays show circumferential fixation at the level of C4-5 using anterior cervical plate and lateral mass screw systems. (B) Barium swallowing study demonstrates good passage of barium in the esophagus. (C) Postoperatively 1 year later, cervical CT showed solid bony fusion at the level of C4-5 bodies.
After the surgery, the patient was mobilized with a collar. Immediately following surgery, dysphagia was improved. The postoperative barium contrast swallow cinematography demonstrated improvement of the filling defect along the pharynx due to removal of the ossified anterior longitudinal ligament (Fig. 2B). The patient was discharged without any complications through postoperative ten days. One year later, cervical CT showed bony fusion at C4-5 bodies and no recurrence of anterior cervical osteophyte (Fig. 2C). The patient did not complain about the dysphagia.

\section{DISCUSSION}

Large anterior cervical osteophytes can produce otolaryngological symptoms such as dysphagia, dysphonia, and foreign body sensation. However, these phenomenon are considered relatively uncommon ${ }^{8)}$. DISH is known as one of the most common causes of anterior cervical hyperosteophytosis induced dysphagia that requires the surgical intervention ${ }^{2,5,8)}$. It is estimated that $3 \%$ of individuals over the age of 40 have DISH and $0.1-6 \%$ of those will develop dysphagia ${ }^{3)}$. The pathogenesis of dysphagia caused by the anterior cervical osteophyte can be explained as a mechanical compression leading to esophageal obstruction, pharyngo-esophageal irritation, and local inflammatory responses resulting in cricopharyngeal spasms and esophageal denervations ${ }^{12)}$. Major diagnostic criteria for DISH include the ossification along the anterolateral aspect of at least four contiguous vertebral bodies, relative preservation of intervertebral disc height, and absence of apophyseal joint ankylosis or sacroiliac joint sclerosis ${ }^{8)}$. In this case, we diagnosed by DISH because C5-T1 vertebral bodies were fused by the anterior bony spur and intervertebral disc height was relatively preserve. And, there was no facet joint ankylosis or sacroiliac joint sclerosis.

While the etiology of DISH may be currently unknown, excessive mechanical stress, hyperlipidemia, and increased levels of insulin and insulin-like growth factor- 1 have been reported as potential predisposing factors, ${ }^{4,6}$. In this present case, cervical vertebral bodies were fused by bridging of the anterior osteophytes that resulted from the ossification of the anterior longitudinal ligament, except C4-5 segment. Therefore, the only mobile C4-5 segment had excessive mechanical stress. Biomechanical studies of human cadaveric cervical spines have shown an increased strain at the adjacent levels after 1-, 2-, and 3-level fixation, suggesting a mechanism for accelerated degeneration of the remaining motion segments after fusion". Presumably, accelerated degenerative changes can result in the anterior osteophyte formation. Our patient had superimposed accelerated degenerative change at the level of C4-5 segment 
on the underlying anterior cervical hyperostosis caused by DISH, resulting in the giant anterior cervical osteophyte.

Dysphagia caused by a cervical osteophyte can be treated with conservative methods, such as dietary modifications, a swallowing therapy and a prescription of non-steroidal anti-inflammatory drugs in the early stage ${ }^{2}$. However, patients may need surgery when these conservative treatments fail or the osteophytes become remarkably large. According to the recent review article, only 35 (20.7\%) of 169 reported cases were treated by nonoperative methods ${ }^{13)}$. The other cases were treated by operative methods and the most common operative technique was resection of the osteophyte without spinal fu$\operatorname{sion}^{13)}$. The osteophytectomy without spinal fusion is advantageous due to no implant-associated complication and less operation time. However, recurrent formation of the osteophyte may occur. The recurrence rate for the osteophyte is unclear, but there are some reports that the presence of postoperative intervertebral mobility was found to be a significant risk factor in the recurrent formation of osteophytes ${ }^{7}$. Specific complications associated with the anterolateral approach for osteophyte resection include vocal cord palsy, Horner syndrome, and esophageal/tracheal perforation or fistula ${ }^{8}$.

In this case, we choose early surgical treatments via the anterior cervical approach because of the relatively young age and extreme large size of the osteophyte. Removal of only the large cervical osteophytes, rather than multilevel anterior resection of DISH, resulted in a rapid postoperative resolution of dysphagia. And our patient had a linear breakage of the ossification at C4-5, which caused instability. To prevent recurrence of osteophyte and to treat the instability, we supposed that the internal fixation was needed. But it seemed that using the anterior cervical plate only induces the implant failure. There was excessive stress loading at the C4-5 segment because all cervical spine segments were fused except C4-5. Therefore, the patient underwent excision of osteophyte and strut grafting at C4-5. The anterior approach was augmented with a posterior stabilization using the lateral mass screws from C3 to C6.

\section{CONCLUSION}

DISH is a common cause of the anterior cervical osteophyte resulting in the progressive dysphagia. Keeping this clinical entity in the differential diagnosis is important in patients with progressive neck stiffness, dysphagia or dysphonia. The surgical treatment of symptomatic anterior cervical osteophyte due to DISH should be considered whether the other segments were fused. If multi-level fusion of the other segments was identified, the surgical strategy must be considered with a solid fusion procedure after the osteophytectomy to prevent segmental instability at the osteophytectomy level and the osteophyte regrowth.

\section{REFERENCES}

1. Aydin E, Akdogan V, Akkuzu B, Kirbas I, Ozgirgin ON: Six cases of Forestier syndrome, a rare cause of dysphagia. Acta Otolaryngologica 126:775-778, 2006

2. Calisaneller T, Ozdemir O, Tosun E, Altinors N: Dysphagia due to diffuse idiopathic skeletal hyperostosis. Acta Neurochirurgica 147:1203-1206; discussion 6, 2005

3. Carlson ML, Archibald DJ, Graner DE, Kasperbauer JL: Surgical management of dysphagia and airway obstruction in patients with prominent ventral cervical osteophytes. Dysphagia 26:34-40, 2011

4. Denko CW, Boja B, Moskowitz RW: Growth promoting peptides in osteoarthritis and diffuse idiopathic skeletal hyperostosisinsulin, insulin-like growth factor-I, growth hormone. The Journal of Rheumatology 21:1725-1730, 1994

5. Ebo D, Goethals L, Bracke P, Mercelis R, De Clerck LS: Dysphagia in a patient with giant osteophytes: case presentation and review of the literature. Clinical Rheumatology 19:70-72, 2000

6. Forestier J, Lagier R: Ankylosing hyperostosis of the spine. Clinical Orthopaedics and Related Research 74:65-83, 1971

7. Miyamoto K, Sugiyama S, Hosoe H, Inuma N, Suzuki Y, Shimizu K: Postsurgical recurrence of osteophytes causing dysphagia in patients with diffuse idiopathic skeletal hyperostosis. European Spine Journal: Official Publication of the European Spine Society, the European Spinal Deformity Society, and the European Section of the Cervical Spine Research Society 18:16521658, 2009

8. Oppenlander ME, Orringer DA, La Marca F, McGillicuddy JE, Sullivan SE, et al: Dysphagia due to anterior cervical hyperosteophytosis. Surgical Neurology 72:266-270; discussion 70-71, 2009

9. Ragab AA, Escarcega AJ, Zdeblick TA: A quantitative analysis of strain at adjacent segments after segmental immobilization of the cervical spine. Journal of Spinal Disorders \& Techniques 19:407-410, 2006

10. Resnick D: Diffuse idiopathic skeletal hyperostosis. AJR. American Journal of Roentgenology 130:588-9, 1978

11. Resnick D, Shaul SR, Robins JM: Diffuse idiopathic skeletal hyperostosis (DISH): Forestier's disease with extraspinal manifestations. Radiology 115:513-524, 1975

12. Srinivas P, George J: Cervical osteoarthropathy: an unusual cause of dysphagia. Age and Ageing 28:321-322, 1999

13. Verlaan JJ, Boswijk PF, de Ru JA, Dhert WJ, Oner FC: Diffuse idiopathic skeletal hyperostosis of the cervical spine: an underestimated cause of dysphagia and airway obstruction. The Spine Journal: Official Journal of the North American Spine Society 11:1058-1067, 2011 